Gina Aracely García-Cedeño; Maira Dolores Vélez-Loor; Carmen Narcisa Franco-Zambrano; Martha Inés Ormaza-Bermello

http://dx.doi.org/10.35381/e.k.v3i5.770

\title{
Educación por competencias: Una posibilidad reordenadora del currículo pensada en emergencia escolar por COVID-19
}

\section{Competency-based education: A reordering possibility of the curriculum designed for school emergencies by COVID-19}

\author{
Gina Aracely García-Cedeño \\ ginita.1983@gmail.com \\ Pontificia Universidad Católica del Ecuador, Portoviejo Manabí \\ Ecuador \\ https://orcid.org/0000-0002-4527-1248 \\ Maira Dolores Vélez-Loor \\ maira.velezloor@yahoo.es \\ Pontificia Universidad Católica del Ecuador, Portoviejo Manabí \\ Ecuador \\ https://orcid.org/0000-0001-7742-8978 \\ Carmen Narcisa Franco-Zambrano \\ carmenfranco4982@gmail.com \\ Pontificia Universidad Católica del Ecuador, Portoviejo Manabí \\ Ecuador \\ https://orcid.org/0000-0002-8290-9634 \\ Martha Inés Ormaza-Bermello \\ marthaormaza09@gmail.com \\ Pontificia Universidad Católica del Ecuador, Portoviejo Manabí \\ Ecuador \\ https://orcid.org/0000-0002-3429-0320
}

Recepción: 18 abril 2020

Revisado: 20 de mayo 2020

Aprobación: 10 junio 2020

Publicación: 19 de junio 2020 
Gina Aracely García-Cedeño; Maira Dolores Vélez-Loor; Carmen Narcisa Franco-Zambrano; Martha Inés Ormaza-Bermello

\title{
RESUMEN
}

El artículo de reflexión tiene el propósito de presentar los resultados del análisis realizado en torno a la educación por competencias como posibilidad reordenadora del currículo en emergencia escolar por COVID-19 en el Ecuador. Se generó una revisión bibliográfica de investigaciones publicadas en revistas arbitradas con la finalidad de conocer el alcance de la educación por competencias y como esta podría contribuir en la conformación de un diseño curricular que reoriente el proceso educativo en el Ecuador. Se persigue que el estudiante se forme para aprender un aprendizaje a lo largo de su vida, de modo auto dirigido y hasta podría considerarse autodidacta, lo cual acompañado de por la investigación, permitirá generar competencias complejas para la construcción de soluciones a los múltiples problemas a los cuales se enfrenta en la cotidianidad. Percibiéndose la necesidad de transcender del modelo cognitivo al de aprendizaje activo, posibilitándose el diseño educativo en base a los pilares de la educación por competencias.

Descriptores: Aprendizaje activo; trabajos prácticos; competencias para la vida; desarrollo de las habilidades. (Palabras tomadas del Tesauro UNESCO).

\begin{abstract}
The reflection article has as a purpose to analyze competency- based learning as a possibility for curricular rearrangement during school emergency by COVID-19 in Ecuador. A bibliographic review of research published in peer-reviewed journals was generated in order to know the scope of the competency- based learning and how this could contribute to the formation of a curricular design that reorients the educational process in Ecuador. It is intended that the students could be formed to get involved in learning throughout their life, in a self-directed way and could even be considered selftaught, which, accompanied by research, will allow generating complex competences for the construction of solutions to the multiple problems faced in everyday life. In this way, they will perceive the need to transcend the cognitive model to that of active learning, making educational design possible based on the pillars of the competency- based learning.
\end{abstract}

Descriptors: Activity learning; practical work (learning method); life skills; skills development. (Words taken from the UNESCO Thesaurus). 
Gina Aracely García-Cedeño; Maira Dolores Vélez-Loor; Carmen Narcisa Franco-Zambrano; Martha Inés Ormaza-Bermello

\section{INTRODUCCIÓN}

Los sistemas educativos convencionales centrados en la modalidad presencial, fueron sorprendidos por la pandemia COVID-19, a causa del virus SARS-CoV-2, por cuanto en modo emergencia han tenido que asumir el período escolar que se encontraba en desarrollo o en otras áreas geográficas recién estaba por iniciar, como es el caso del régimen costa en el Ecuador, por lo cual se ha tenido que redefinir a la atención en el hogar de los estudiantes.

Esto ha implicado trasladar parte de los temas planificados en modalidad presencial a la virtual, diseñar nuevos en función de concordar con el enfoque TIC y los diversos recursos tecnológicos por donde se puede promover el aprendizaje, sin embargo, quienes habitualmente ejercían labores pedagógicas en modalidad presencial y hacían uso ocasional de las TIC en fomentar el aprendizaje, se han visto sorprendido por tener que asumir una nueva modalidad educativa.

Esto genera la incertidumbre sí se está realizando un trabajo educativo en conformidad al enfoque de las TIC o simplemente se está asumiendo una postura de resolver sobre la marcha, con la intención de que los estudiantes no pierdan el período académico, así como por parte de los gobiernos justificar el pago al sector educativo. En fin, son circunstancias que deben ser atendidas con la importancia del caso, para lo cual, se toma el enfoque por competencias como una opción para promover propuestas educativas en favor de ordenar la incertidumbre generada a causa de la pandemia por COVID-19, en este sentido, Robles-Haros \& Estévez-Nenninger (2015), plantea que:

Es necesario agregar que el enfoque por competencias no surge de la nada, surge de un proyecto muy bien estructurado y en consenso con muchos países, tanto de la Unión Europea, como de América Latina, por ello se debe preparar a la comunidad educativa en cuanto al uso e implementación de los nuevos enfoques o modelos de enseñanza-aprendizaje, buscando referentes de éxito, para lograr el interés del personal docente al constatar la utilidad y beneficios que tendrá en el estudiantado (p. 10). 
Gina Aracely García-Cedeño; Maira Dolores Vélez-Loor; Carmen Narcisa Franco-Zambrano; Martha Inés Ormaza-Bermello

Lo cual implica que este enfoque es diseñado con intenciones particulares de educar para promover un aprendizaje alternativo al conocido como convencional o cognitivo basado en la aprehensión de conocimientos, en la educación por competencias se trata de constituir el aula en un ambiente donde se aprende simulando acciones de la vida real, donde el estudiante tiene la oportunidad de demostrar habilidades, destrezas, diseñar, innovar, actuar, entre otras cualidades, integrando conocimientos y operaciones hasta constituir una aptitud que le permita desempeñarse fuera del ámbito escolar en razón de brindar respuestas a múltiples necesidades particulares y sociales.

En complemento, Díaz-Díaz (2017), destaca que el currículo por competencias propicia "el desarrollo de las capacidades complejas que permiten a los estudiantes pensar y actuar en diversos ámbitos". La competencia también se entiende como "una combinación entre destrezas, habilidades y conocimientos necesarios para desempeñar una tarea específica" (p. 1), por lo cual, ha sido asumido por empresas para la formación permanente del talento humano, así se puede dilucidar que este enfoque tiene relación con la formación técnica, así como la educación dual, por lo cual, se hace versátil para ser aplicado en diversas realidades y contextos sociales en función de responder a sus necesidades.

La educación por competencias se dinamiza sobre la base de promover el aprender a aprender, aprender a hacer, aprender a pensar, aprender a comunicar, aprender a vivir juntos (Roegiers, 2016), de ese modo, se abre la posibilidad de diseñar currículos en donde se promueve un aprendizaje en cada una de las áreas mencionadas, por lo cual, se educa con una visión compleja de la realidad en función de gestionar acciones favorables para que el estudiante pueda transversalmente actuar en función de lograr metas propuestas en la planificación académica, de ese modo, (Germain \& Pérez-Rico, 2014), indican que el abordaje por competencias contribuye al estudiante se empodere del conocimiento, mediante la indagación permanente a las múltiples inquietudes e interrogantes que surgen al desarrollar un determinado proyecto. 
Gina Aracely García-Cedeño; Maira Dolores Vélez-Loor; Carmen Narcisa Franco-Zambrano; Martha Inés Ormaza-Bermello

El aprendizaje por competencias se centra en el estudiante, promoviendo cambios en su concepción de percibir la realidad, así como su protagonismo educativo (Burgos-Briones, Pico-Barreiro \& Vélez-Zambrano, 2019), lo cual obliga al docente a promover nuevas perspectivas de planificación educativa, por cuanto al proponer el enfoque por competencias, este se distancia de la modalidad convencional de educación en cuanto su concepción filosófica curricular (Vallejo-Valdivieso, et al, 2019).

Desde lo abordado, se concibe el currículo como una oportunidad para construir cambios sociales (Garzón-Daza, 2017), siendo necesario que el docente conciba el currículo como un marco para direccionar la educación con base a lineamientos políticos, pedagógicos, epistemológicos, focalizado hacia un accionar transformador de la realidad y no como un mero documento técnico (Guanipa-Gotopo, 2018).

Por otro lado, (Casanova-Romero, et al., 2018), indica que en los países latinoamericanos donde se dice trabajar con el enfoque por competencias, no se evidenció un sistema de evaluación certificado por competencias, lo cual es fundamental para conocer no solo que se evalúa, sino que se aprende y como se aprende, por cuanto al ser un aprendizaje centrado en lo demostrativo se hace necesario contar con rubricas para identificar los logros por parte de los participantes.

En el caso del Ecuador, se ha promovido la certificación por competencias laborales (Servicio Ecuatoriano de Capacitación Profesional -SECAP, 2016), lo cual ha permitido que las personas que aprueban las pruebas teóricas - prácticas sean acreditadas con credenciales para ejercer un determinado oficio o trabajo, lo cual se constituye en un antecedente inmediato a tener en cuenta en el sistema educativo nacional. Otro referente es la educación dual - tecnológica que ha venido impulsándose en los institutos tecnológicos del país en conjunto con las empresas, lo cual a juicio de (Carvajal, Romero \& Álvarez, 2017), consideran que esta podría contribuir a una mejor preparación de los estudiantes para su inserción al mercado laboral, por cuanto reciben el acompañamiento de un tutor empresarial (Zavala-Alcívar, García-Loor \& López-Figueroa, 2019). 
Gina Aracely García-Cedeño; Maira Dolores Vélez-Loor; Carmen Narcisa Franco-Zambrano; Martha Inés Ormaza-Bermello

Lo planteado hace pensar en la posibilidad de proyectar el enfoque por competencias como una alternativa pedagógica y reorientar el currículo a la luz de la pandemia por COVID-19 que ha obligado asumir de emergencia lo virtual como alternativa para cumplir con el periodo académico en el Ecuador, por lo que se abre la posibilidad de diseñar un currículo basado en competencias para formar sobre las nuevas necesidades sociales enfocadas en lo digital, para lo cual, se podría desde el ámbito escolar, educar no solo en procesos cognitivos, sino, operacionales en base a los requerimientos de una sociedad global sustentada en la internet como nueva fuente de empleo.

A razón de todo lo planteado, concretamente se pretende presentar las reflexiones construidas en torno al análisis realizado al enfoque de la educación por competencias como posibilidad reordenadora del currículo en emergencia escolar por COVID-19 en el Ecuador.

\section{MÉTODO}

Para el desarrollo de la investigación se generó una revisión bibliográfica y se aplicó el análisis de contenido como técnicas de recopilación y procesamiento de información documental de investigaciones publicadas en revistas arbitradas con la finalidad de conocer el alcance de la educación por competencias y como esta podría contribuir a repensar un diseño curricular que reoriente el proceso educativo en el Ecuador. Ordenándose así, áreas de trabajo por medio de la cual, el estudiante no solo tenga la oportunidad de desarrollar un proceso cognitivo, sino también, el desarrollo de destrezas, capacidades, habilidades, desde el punto de vista lógico - matemático, espacial lingüístico, informática educativa, procesamiento de operaciones para configurar operaciones en internet con fines de interconectarse a la red de teletrabajo de la era digital. 
Gina Aracely García-Cedeño; Maira Dolores Vélez-Loor; Carmen Narcisa Franco-Zambrano; Martha Inés Ormaza-Bermello

\section{RESULTADOS}

\section{Educación por competencias}

La visión de educación por competencias, requiere generar una visión filosófica de comprensión de la realidad educativa desde una vertiente que promueva tanto en el docente como estudiantes, las condiciones de evaluación de los aprendizajes en consonancia con este enfoque (Vidal-Ledo, et al., 2016), por lo que la aplicación esta corriente curricular debe ser producto de una fuerte reflexión y ensayo previo, de ese modo, se evita la posibilidad de aplicar experiencias que no se corresponden con la esencia de este modelo educativo.

En complemento, (Bicocca-Gino, 2017), comenta que el enfoque basado por competencias ha recibido críticas por su vertiente economicista de formación, siendo necesario no descuidar la competencia referida a lectura crítica y ético de la realidad social, por cuanto esto le permite al estudiante, no solo capacitarse, sino, formarse integralmente, lo cual distanciaría a este modelo de una mero proceso instruccional de adquisición del conocimiento y procedimientos para responder a una determinada actividad laboral, teniendo vigencia el planteamiento de (Camperos-Camero, 2008), al indicar:

En cuanto a la evaluación en sí misma, si no hay un trabajo compartido y participativo de carácter reflexivo que lleve a consensos entre los académicos (profesores) sobre qué competencias deben adquirir los alumnos y qué debe evidenciarse cómo logro, no se podrá concretar un proceso de evaluación pedagógicamente válido (p. 813).

Lo que deja en evidencia que este enfoque, primordialmente se sustenta en el trabajo cooperativo y participativo de los participantes, con lo cual, se articula una construcción de saberes en complejidad de acciones para lograr un determinado fin. En complemento, (Ríos-Muñoz \& Herrera-Araya, 2017), consideran que la evaluación debe ser sustentada desde un proceso formativo en prosecución de fomentar un aprendizaje autorregulado y autoaprendizaje, lo cual promueve el pilar de aprender a aprender, así mismo (Bermúdez-Bedoya, 2016), considera implementar programas formativos para promover 
Gina Aracely García-Cedeño; Maira Dolores Vélez-Loor; Carmen Narcisa Franco-Zambrano; Martha Inés Ormaza-Bermello

cambios significativos en docentes y estudiantes con la finalidad de asumir con eficacia el aprender a aprender.

Por otro lado, Pulido-Cortés \& Gómez-Gómez (2017), comentan que "el aprender está situado en las estructuras de pensamiento, el proceso, la actividad y la posibilidad de confrontación de los conocimientos cotidianos con los científicos" (p. 9), así se conforma un proceso articulado donde se promueve la construcción de saberes en apoyo de las tecnologías y otros recursos en concordancia a las metas planteadas por el equipo de trabajo. (Núñez-Castillo, et al., 2017), destacan que la comprobación por parte de los estudiantes confirma la visión de aprender a aprender.

El aprender a hacer, se proyecta como un pilar por el cual los estudiantes tienen la oportunidad de integrar teoría con práctica, siendo esto uno de los retos fundamentales en la educación de actual (Arteaga-Valdés, Armada-Arteaga \& Del-Sol-Martínez, 2016). De igual manera (León-Salas, 2015), enfatiza que se aprende haciendo con el acompañamiento del docente, el cual hace el rol de tutor o asesor para construir en equipo un determinado proyectado, en función de metas establecidas previamente.

El aprender a comunicar, es transversal por cuanto esta genera la posibilidad de trabajar la introspección como método para la búsqueda del ser, aprendiendo a la comunicación interna, proceso que contribuye a la reflexión metódica con la finalidad de poder generar compromiso con el aprendizaje, así como con situaciones de la vida diaria (NarváezMontoya, 2019), por lo cual, debe ser complementada con una comunicación asertiva que permita comunicarse exteriormente, por cuanto esta contribuye a comunicar con efectividad lo que realmente se pretende comunicar (Cañas \& Hernández, 2019), siendo indispensable hoy día, comunicarse por medio de las TIC, lo cual podría catalogarse como una comunicación basada en las tecnologías, lo cual contribuye al mejoramiento de las capacidades cognitivas (Vértiz-Osores, et al., 2019).

El aprender a pensar es un reto de la educación actual y venidera por cuanto se corre el riesgo en medio de asumir la virtualidad para solventar el periodo académico en medio 
Gina Aracely García-Cedeño; Maira Dolores Vélez-Loor; Carmen Narcisa Franco-Zambrano; Martha Inés Ormaza-Bermello

del COVID-19, como mera instrumentalidad, dejando de lado lo complejo de pensar para la reflexión (Maldonado, 2015), siendo que en el enfoque por competencias, se debe abordar como tramo transversal para promover el aprendizaje significativo en los estudiantes.

Por otro lado, el aprender a convivir no solo con los semejantes, sino, con el entorno ambiental, se fundamenta en la posibilidad de concretar una mentalidad ecológica en pro de una ciudadanía donde interviene la ética como eje de las interrelaciones sociales (Fierro-Evans \& Carbajal-Padilla, 2019), en complemento (Calderón, 2018), focaliza la filosofía de la alteridad como una doctrina que permite vivir éticamente en equilibrio de lo social, siendo posibilitadora de encuentro con el otro, en base a relaciones de reconocimiento de las capacidades y potencialidades, lo cual, contribuye a una mejor educación en donde se promueve el crecimiento integral de la persona.

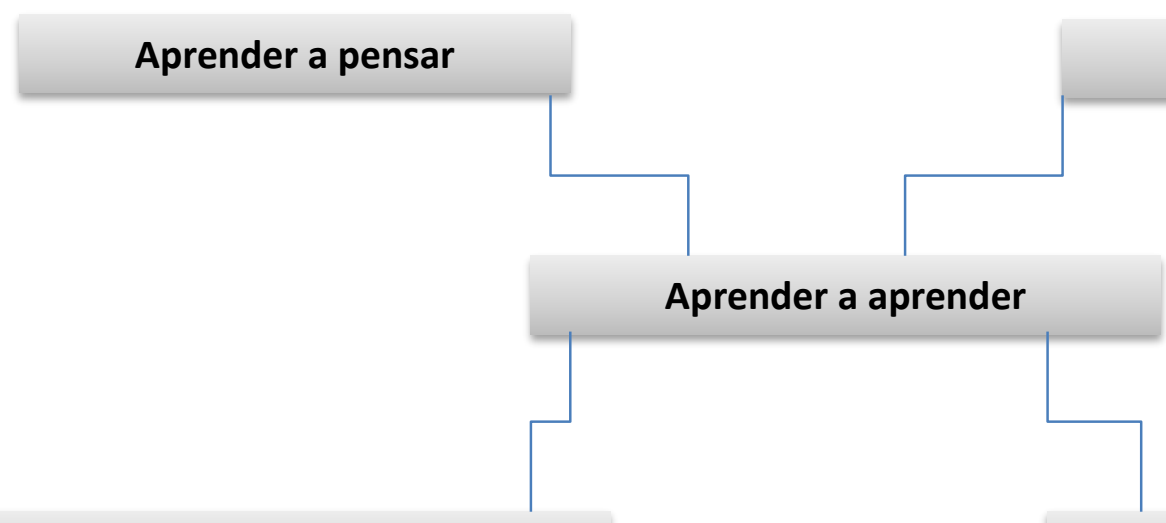

Aprender a vivir juntos

\section{Aprender a hacer}

Aprender a comunicar

Figura 1. Pilares de la educación a partir de Roegiers, 2016.

Fuente: A partir de Roegiers (2016) 
Gina Aracely García-Cedeño; Maira Dolores Vélez-Loor; Carmen Narcisa Franco-Zambrano; Martha Inés Ormaza-Bermello

En este sentido, dentro del enfoque por competencias, se pueden asumir diversas metodologías, entre los cuales se encuentra aprendizaje por proyectos, aprendizaje basado en problemas, los cuales se focalizan en la centralidad del estudiante como protagonista del proceso, siendo el docente un asesor que guía, orienta, acompaña, en la construcción de saberes para la demostración del conocimiento por parte del estudiante, así se genera un amplio espectro de posibilidades pedagógicas para el diseño de un currículo que en la actualidad debe responder a una sociedad basada en lo digital.
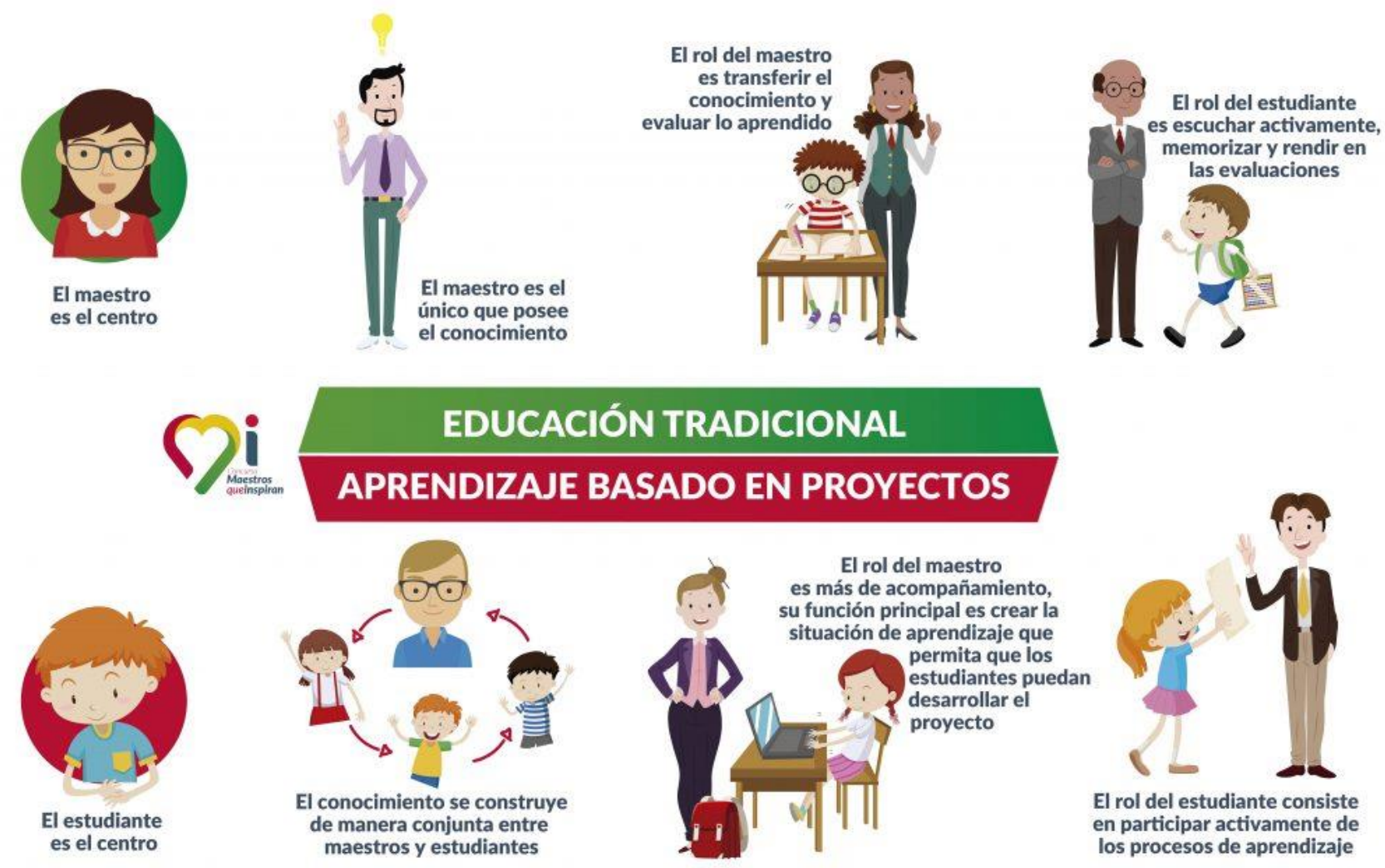

Figura 2. Iconografía de modelos de aprendizaje.

Fuente: Universidad Nacional Abierta y a Distancia. (2011). Lección 58. 
Gina Aracely García-Cedeño; Maira Dolores Vélez-Loor; Carmen Narcisa Franco-Zambrano; Martha Inés Ormaza-Bermello

Se evidencia en este sentido, que el enfoque por competencias, desarrollado por alguna de las metodologías activas de aprendizaje, persigue que el estudiante se forme para aprender un aprendizaje a lo largo de su vida, de modo auto dirigido y hasta podría considerarse autodidacta, lo cual acompañado de por la investigación, permitirá generar competencias complejas para la construcción de soluciones a los múltiples problemas a los cuales se enfrenta en la cotidianidad.

\section{CONCLUSION}

El enfoque de educación por competencias se constituye en una alternativa curricular para reorientar el sistema educativo ecuatoriano en favor de formar en función de formar no solo por la emergencia del COVID-19; sino, de superar las debilidades integrales que han sido develadas en la sociedad, siendo indispensable educar para convivir en respeto mutuo, pero además para responder a una comunidad global basada en lo tecnológico y digital, lo cual conlleva a reducir el campo de empleo tradicional, situación que conlleva a reflexionar sobre el rol de la educación, percibiéndose la necesidad de transcender del modelo cognitivo al de aprendizaje activo, posibilitándose el diseño educativo en base a los pilares de la educación por competencias, siendo necesario sensibilizar y formar para transcender el estatus quo socio educativo anclado a la transmisión de contenidos, de ese modo, se proyecta recopilar información empírica en una segunda fase de investigación con la intención de presentar una propuesta curricular basada en el enfoque por competencias.

\section{FINANCIAMIENTO}

No monetario 
Gina Aracely García-Cedeño; Maira Dolores Vélez-Loor; Carmen Narcisa Franco-Zambrano; Martha Inés Ormaza-Bermello

\section{AGRADECIMIENTOS}

A la Pontificia Universidad Católica del Ecuador, Extensión Manabí, Portoviejo y al Doctor Patricio Alfredo Vallejo-Valdivieso por motivar la construcción de este artículo desde nuestra experiencia educativa.

\section{REFERENCIAS}

Arteaga-Valdés, E, Armada-Arteaga, L, \& Del-Sol-Martínez, J. (2016). La enseñanza de las ciencias en el nuevo milenio. Retos y sugerencias. Revista Universidad y Sociedad, 8(1), 169-176. Recuperado de https://n9.cl/7mku

Bermúdez-Bedoya, J., García-Castro, L., Rivera-González, R., \& Aristizábal-Montes, M. (2016). Aprender a aprender en un modelo de competencias laborales. Zona Próxima, O(25). Recuperado de https://n9.cl/7ng55

Bicocca-Gino, R. (2017). Análisis crítico-filosófico de las potencialidades educativas de la enseñanza basada en competencias. Educación y Educadores, 20(2), 267-281. Recuperado de https://url2.cl/TaBVq

Burgos-Briones, J., Pico-Barreiro, L., \& Vélez-Zambrano, G. (2019). El maestro y la educación sostenible 2030. [The teacher and sustainable education 2030]. CIENCIAMATRIA, 6(10), 609-624. https://doi.org/10.35381/cm.v6i10.241

Cañas, D., \& Hernández, J. (2019). Comunicación asertiva en profesores: diagnóstico y propuesta educativa. Praxis \& Saber, 10(24), 143-165. https://doi.org/10.19053/22160159.v10.n25.2019.8936

Casanova-Romero, I., Canquiz-Rincón, L., Paredes-Chacín, I., \& Inciarte-González, A. (2018). Visión general del enfoque por competencias en Latinoamérica. [Overview of the competence-based approach in Latin America]. Revista de Ciencias Sociales. 24(4), 114-125. Recuperado de https://n9.cl/e708

Carvajal, R, Romero, A, \& Álvarez, G. (2017). Estrategia para Contribuir a la Implementación de la Formación Dual de los Profesionales de Ciencias Empresariales en las Pequeñas y Medianas Empresas de la Provincia Tungurahua, Ecuador. Formación universitaria, 10(5), 29-40. https://dx.doi.org/10.4067/S0718-50062017000500004 
Gina Aracely García-Cedeño; Maira Dolores Vélez-Loor; Carmen Narcisa Franco-Zambrano; Martha Inés Ormaza-Bermello

Camperos-Camero, M. (2008). La evaluación por competencias, mitos, peligros y desafíos. Educere, 12(43), 805-814. Recuperado de https://url2.cl/wCTma

Calderón, A. (2018). La convivencia social entre la ética y el pluralismo: "Una perspectiva desde Emmanuel Lévinas". Revista Electrónica "Actualidades Investigativas en Educación", 18(2), 1-17. https://doi.org/10.15517/aie.v18i2.33165

Díaz-Díaz, H. (2017). Las claves del Nuevo Currículo: El enfoque por competencias. Recuperado de https://n9.cl/gzn3

Fierro-Evans, C, \& Carbajal-Padilla, P. (2019). Convivencia Escolar: Una revisión del concepto. Psicoperspectivas, 18(1), 9-27. Epub 15 de marzo de 2019.https://dx.doi.org/10.5027/psicoperspectivas-vol18-issue1-fulltext-1486

Garzón-Daza, C. (2017). Implicaciones curriculares del enfoque por competencias en educación superior. Revista Boletín Redipe, 4(9), 52 - 76. Recuperado de https://n9.cl/5ihw

Germain, F., \& Pérez-Rico, C. (2014). La educación por competencias como medio para facilitar la toma de control del aprendizaje por el estudiante. FEM: Revista de la Fundación Educación Médica, 17(1), 11-19. https://dx.doi.org/10.4321/S2014$\underline{98322014000100004}$

Guanipa-Gotopo, C. (2018). Aproximación Teórica-epistemológica de la Gestión Curricular, en el contexto de las Universidades Politécnicas Territoriales. Revista Arbitrada Interdisciplinaria Koinonía, 3(5), 27-52. Recuperado de https://n9.cl/uhs $4 \mathrm{w}$

León-Salas, A. (2015). Aprender haciendo: Uso de una estrategia didáctica en un curso avanzado de la carrera de Farmacia de la Universidad de Costa Rica. Revista Educación, 39 (2), 105-113. Recuperado de https://n9.cl/47as

Maldonado, C. (2015). Pensar la complejidad, pensar como síntesis. Cinta de moebio, (54), 313-324. https://dx.doi.org/10.4067/S0717-554X2015000300008

Narváez-Montoya, A. (2019). Comunicación educativa, educomunicación y educación mediática: una propuesta de investigación y formación desde un enfoque culturalista.

Palabra

Clave, 22(3), e22311. https://dx.doi.org/10.5294/pacla.2019.22.3.11 
Gina Aracely García-Cedeño; Maira Dolores Vélez-Loor; Carmen Narcisa Franco-Zambrano; Martha Inés Ormaza-Bermello

Núñez-Castillo, C, Quinzan-King, A, Valle-Castañeda, W, \& González-Márquez, M. (2017). "Aprender a aprender" en la universidad cubana actual. Revista de Ciencias Médicas de Pinar del Río, 21(6), 140-147. Recuperado de https://n9.cl/c15sq

Pulido-Cortés, O., \& Gómez-Gómez, L. (2017). Del enseñar y el aprender. Praxis y Saber, 8 (18), 9-14. https://dx.doi.org/10.19053/22160159.v8.n18.2017.7252

Ríos-Muñoz, D, \& Herrera-Araya, D. (2017). Los desafíos de la evaluación por competencias en el ámbito educativo. Educação e Pesquisa, 43(4), 1073-1086. https://doi.org/10.1590/s1678-4634201706164230

Robles-Haros, B., \& Estévez-Nenninger, E. (2015). Competence Approach: Educational Problems Faced by Teachers. Revista Electrónica Educare, 20(1), 1-12. https://doi.org/10.15359/ree.20-1.25

Roegiers, X. (2016). Marco conceptual para la evaluación de competencias. Oficina Internacional de Educación de la UNESCO. Recuperado de https://n9.cl/1z1a

Servicio Ecuatoriano de Capacitación Profesional -SECAP (2016). Reglamento de capacitación profesional y certificación de personas. Recuperado de https://n9.cl/ukmip

Universidad Nacional Abierta y a Distancia. (2011). Lección 58. Recuperado de https://n9.cl/1653

Vallejo-Valdivieso, P., Zambrano-Pincay, G., Vallejo-Pilligua, P., \& Bravo-Cedeño, G. (2019). Modelos de planificación educativa y diversidad en aulas de clases. [Models of educational planning and diversity in classrooms]. CIENCIAMATRIA, 5(9), 302-315. https://doi.org/10.35381/cm.v6i10.149

Vértiz-Osores, R, Pérez-Saavedra, S, Faustino-Sánchez, M, Vértiz-Osores, J, \& Alain, L.. (2019). Information and Communication Technology in Primary School Students within the Framework of Inclusive Education at a Special Basic Education Center. Propósitos y Representaciones, 7(1), 8394. https://dx.doi.org/10.20511/pyr2019.v7n1.266

Vidal-Ledo, M, Salas-Perea, R, Fernández-Oliva, B, \& García-Meriño, A. (2016). Educación basada en competencias. Educación Médica Superior, 30(1) Recuperado de https://url2.cl/UXJXX 
EPISTEME KOINONIA

Revista Electrónica de Ciencias de la Educación, Humanidades, Artes y Bellas Artes

Año III. Vol III. N5. Enero - Junio, 2020

Hecho el depósito de Ley: FA2018000022

ISSN: 2665-0282

FUNDACIÓN KOINONIA (F.K).

Santa Ana de Coro, Venezuela

Gina Aracely García-Cedeño; Maira Dolores Vélez-Loor; Carmen Narcisa Franco-Zambrano; Martha Inés Ormaza-Bermello

Zavala-Alcívar, A., García-Loor, G., \& López-Figueroa, V. (2019). Inserción laboral de la formación dual técnica y tecnológica en el Ecuador. CIENCIAMATRIA, 5(1), 103117. https://doi.org/10.35381/cm.v5i1.252

@2020 por el autor. Este artículo es de acceso abierto y distribuido según los términos y condiciones de la licencia Creative Commons Atribución-NoComercial-Compartirlgual 4.0 Internacional (CC BY-NC-SA 4.0)

(https://creativecommons.org/licenses/by-nc-sa/4.0/). 